\title{
Editorial: Adiós al rastreo de cáncer de próstata con las técnicas actuales
}

\author{
Goodbye to prostate cancer screening with the current technologies
}

De acuerdo al dictamen de un importante organismo gubernamental de cuidado preventivos los hombres sanos no deberían hacerse más un antígeno prostático específico (PSA por su sigla en inglés) como rastreo ("screening") del cáncer de próstata ya que la prueba no salva vidas y conduce frecuentemente a más pruebas y tratamientos innecesarios que causan dolor, impotencia e incontinencia a muchos.

En mayo de 2012 la fuerza de tareas estadounidense de prácticas preventivas (USPSTF) finalmente se expidió en contra del rastreo con PSA para el cáncer de próstata ${ }^{1}$ en hombres de la población general independientemente de su edad (recomendación $\mathrm{D}^{2,3}$ ). Esta recomendación no incluye el uso del PSA para la vigilancia después del diagnóstico o el tratamiento del cáncer de próstata, lo cual no fue evaluado por la USPSTF. Esta recomendación ha suscitado tal polémica tanto en el mundo científico como en el periodístico ${ }^{4}$ que llevó a Michael LeFevre, co-director de la USPSTF, a pronunciarse:

"El cáncer de próstata es un problema de salud grave que afecta a miles de hombres y sus familias. Pero antes de obtener una prueba de PSA, todos los hombres tienen derecho a saber lo que la ciencia nos dice acerca de la prueba de PSA: hay un beneficio potencial muy pequeño y significativos daños potenciales. Animamos a los médicos a considerar esta evidencia, y no rastrear a sus pacientes con una prueba de PSA a menos que el individuo que está siendo evaluado entienda lo que se sabe acerca de la prueba de PSA y tome la decisión personal de que aun una pequeña posibilidad de beneficio supere (para él) los riesgos de daños ya conocidos".

En octubre de 2011, la USPSTF publicó, para someterlo a comentarios del público, el borrador de proyecto de recomendación sobre rastreo del cáncer de próstata. Desde entonces, la USPSTF recibió numerosos comentarios y revisó la evidencia más actualizada a mayo del 2012, y tras un extenso y profundo debate llegó a la conclusión de que muchos hombres se ven perjudicados como consecuencia del rastreo del cáncer de próstata y pocos, o ninguno, son beneficiados. Hasta que estén disponibles una mejor prueba y mejores opciones terapéuticas, la USPSTF se ha expedido contra el rastreo de cáncer de próstata.

La decisión fue adoptada sólo después de un amplio examen y debate serio. Se describen aquí estimaciones de posibles beneficios y daños de los hombres de 55 a 69 evaluadas con una prueba de PSA cada 1 a 4 años durante 10 años.

\section{Posible beneficio de la detección temprana mediante el} rastreo

La reducción en las muertes por cáncer de próstata mediante el PSA es a lo sumo pequeña.

Un gran estudio realizado en EE.UU. no mostró ningún beneficio de la detección y otro europeo que encontró el beneficio más alto reportado sugiere que el rastreo evitaría, como máximo, una muerte por cáncer de próstata cada 1.000 hombres rastreados (El riesgo a 10 años de por morir de cáncer de próstata es de 5 en 1.000 sin rastreo y de 4 a 5 en 1.000 con rastreo).

\section{Daños esperados de la detección}

La mayoría de los cánceres de próstata que se encuentran por el PSA son de crecimiento lento, no amenazan la vida, y no causarán daños durante toda la vida de un hombre. Sin embar- go, actualmente no hay manera de determinar qué tipos de cáncer es probable que amenacen la salud de un hombre y cuáles no. Por ende, casi todos los hombres con cáncer de próstata detectados por el PSA optan por recibir tratamiento. La prueba de detección del PSA a menudo sugiere que el cáncer de próstata puede estar presente cuando no hay cáncer ("falso positivo"). Estos resultados generan preocupación y ansiedad y puede resultar en pruebas de seguimiento y procedimientos, tales como biopsias innecesarias. Las biopsias pueden causar en un $33 \%$ daños tales como fiebre, infección, hemorragia, problemas urinarios y dolor. Incluso un $1 \%$ de hombres tiene que ser hospitalizado a causa de estas complicaciones.

Alcanzado el diagnóstico, las estrategias de manejo para el cáncer de próstata localizado son: conducta expectante, vigilancia activa, cirugía y/o radioterapia. Además de las complicaciones asociadas al rastreo y a la cascada diagnóstica que este puede generar, los daños secundarios al tratamiento del cáncer de próstata detectados por rastreo son claros y pueden ser graves.

Por cada 1.000 hombres rastreados con PSA:

- 100 a 120 tienen al menos un resultado falso positivo.

- Diez hombres se internan a causa de complicaciones de la biopsia prostática.

- Dos hombres experimentan un evento cardiovascular grave, como infarto de miocardio, debido al tratamiento.

- Un hombre desarrolla trombosis venosa grave en los miembros inferiores o los pulmones debido al tratamiento.

- 29 hombres desarrollan disfunción eréctil debido al tratamiento. - 18 hombres desarrollan incontinencia urinaria debido al tratamiento.

Además, por cada 3.000 hombres que se someten a tamizaje con la prueba de PSA:

- Un hombre muere debido a complicaciones del tratamiento quirúrgico.

Estos datos reflejan una distribución de las intervenciones posterior al diagnóstico del $60 \%$ para los tratamientos quirúrgicos, $30 \%$ para radioterapia y $10 \%$ para observación (ver abajo para más detalles sobre los supuestos y referencias). No se contemplan además otros daños de la radiación, tales como la enteritis actínica. Es importante entender que muchos de estos hombres en realidad no necesitaban tratamiento, ya que su cáncer no habría crecido ni habría causado problemas de salud, incluso sin tratamiento. A este fenómenos se lo denomina "sobretratamiento".

\section{Preguntas frecuentes con respecto al rastreo con PSA}

- ¿Qué significa esta recomendación para el hombre común? Hasta que no tengamos mejores pruebas y mejores opciones terapéuticas, la USPSTF, basada en una exhaustiva revisión de la literatura, recomienda que los hombres no se hagan el PSA para detectar el cáncer de próstata. Hacerlo o no es una decisión que cada hombre debe tomar en base a sus propios valores y preferencias una vez que entienda cabalmente los riesgos y beneficios que conlleva. Si balance no es lo suficientemente claro es recomendable que cada persona discuta el tema en profundidad con su médico.

- ¿Cómo debe decidir el paciente si hacerse o no un PSA?

El paciente debe saber lo que dice la ciencia sobre la detección del PSA: existe un pequeño beneficio potencial y existen impor-

Servicio de Medicina Familiar y Comunitaria del Hospital Italiano de Buenos Aires agustin.ciapponi@hospitalitaliano.org.ar 
tantes daños potenciales. Pero también debe pensar acerca de sus creencias y preferencias personales para el cuidado de la salud. Sopesar los beneficios y daños potenciales de la prueba de PSA y decidir qué es más importante para él. Si existe la posibilidad, por pequeña que sea, de evitar la muerte por cáncer de próstata quizás sea más importante para él que el riesgo de daños innecesarios y, en ese caso, hacerse el PSA puede ser la decisión correcta. El médico debe ayudarlo a entender la evidencia científica y a tomar una decisión que refleje sus valores y preferencias ${ }^{5}$.

- ¿A quién se aplica esta recomendación contraria a realizarse el rastreo de cáncer de próstata?

Esta recomendación es para los hombres adultos de todas las edades. No se aplica a los hombres que han sido diagnosticados o estén recibiendo tratamiento para el cáncer de próstata. Los hombres mayores, afroamericanos o con historial familiar de cáncer de próstata tienen mas riesgo de desarrollar cáncer de próstata. La actual evidencia disponible no nos permite saber con certeza si el balance entre beneficios y daños es muy diferente en estos casos, pero sí que justifica un cuidadoso diálogo médico-paciente para facilitar la toma de decisión ${ }^{4}$.

- El número de muertes por cáncer de próstata ha disminuido en los últimos años. ¿Por qué es esto? ¿Puede que esta disminución se atribuya al rastreo?

Por razones desconocidas, la tasa de mortalidad por cáncer de próstata ha estado subiendo desde hace dos décadas hasta alrededor de 1992, y luego comenzó a caer. La prueba de PSA no se convirtió en una práctica clínica generalizada hasta mediados de la década de 1990. Debido a que el cáncer de próstata es una enfermedad de crecimiento lento, los grandes ensayos clínicos han demostrado que una reducción en las muertes debidas al rastreo no se evidenciará hasta siete a diez años después del comienzo del rastreo. Como tal, resulta improbable que la inversión de la tendencia creciente de las tasas de mortalidad por cáncer de próstata se relacione al rastreo. Una explicación más plausible es la mejora en el cuidado de la salud en general y específicamente en el tratamiento de este tipo de cáncer. De hecho otros tipos de cánceres para los que no se realiza frecuentemente el rastreo también mostraron descensos en las tasas de mortalidad en el mismo período.

- ¿Qué respondió la USPSTF a los comentarios públicos recibidos sobre el proyecto de recomendación contraria a realizarse el rastreo de cáncer de próstata?

- Una "recomendación D" no significa que los médicos y los pacientes no puedan hablar del examen para el cáncer de próstata. Por el contrario se hace hincapié en que los médicos y pacientes deben hablar sobre los posibles beneficios y daños, así como las preferencias individuales de cada paciente, antes de decidirse a iniciar o continuar el rastreo.

- Se agregó una tabla indicando los riesgos y beneficios del cribado.

- El grupo de trabajo se compuso de 16 miembros voluntarios expertos en la prevención y la medicina basada en la evidencia. Casi todos prestan en la actualidad cuidados a pacientes de atención primaria como médicos de familia, médicos generales, internistas, enfermeras, gineco-obstetras, médicos laborales y pediatras.

- El grupo de trabajo no incluyó urólogos, sin embargo, expertos de esta especialidad brindaron revisión de pares de la revisión sistemática realizada por la USPSTF.

- ¿Qué recomiendan otras organizaciones acerca del rastreo del cáncer de próstata? ¿Colaboraron con el grupo de trabajo?

El grupo de trabajo de la USPSTF aprecia los aportes de las organizaciones interesadas y del público general, y es por eso que junto a cada publicación del proyecto de recomendación se encuentra disponible en su página web la opción de realizar comentarios afines.

La USPSTF, como panel independiente, no colaboro con otras organizaciones para hacer esta recomendación, sin embargo, es consciente de las recomendaciones de otros grupos e incluye una sección en la declaración de la recomendación final sobre las recomendaciones de otras organizaciones ${ }^{6}$.

La Asociación Urológica Americana ha dado marcha atrás con su recomendación de rastreo de rutina por otra de discutir con el paciente los riesgos y los beneficios del rastreo.

\section{Reflexiones finales}

No resultan sorprendentes las nuevas recomendaciones emitidas por la USPSTF. Las evidencias analizadas en editoriales anteriores son totalmente consistentes con las mismas ${ }^{7,8}$. No existían ya más razones para demorarlas a no ser por la fuerte polémica que como era previsible ocurrió.

El jugador de béisbol Joe Torre, el financista Michael Milken y Rudolph W. Giuliani, ex alcalde de Nueva York, se encuentran entre las decenas de miles de hombres que creen que una prueba de PSA les salvó la vida ${ }^{4}$. Es difícil explicar a personas con tal grado de involucramiento que muchos de ellos podrían haberse evitado la conmoción del diagnóstico y sus consecuencias, y que sus vidas ni siquiera hubieran atravesado sobresaltos de padecer, sin saberlo, el cáncer de próstata más común que es el de lento crecimiento. Estas personas lucharán contra la recomendación y es comprensible que lo hagan.

Para trazar un paralelismo social, tampoco es bueno legislar en cuestiones de seguridad, basado en un hecho puntual por doloroso que sea.

El problema se presenta a veces por dos visiones contrapuestas acerca de las intervenciones preventivas. Por un lado están aquellos, a veces denominados "evangelistas", que sostienen que ante la mínima posibilidad de beneficio, éstas deberían ofrecerse activamente a los pacientes. Por el otro están quienes afirman que una intervención sólo debiera ofrecerse ante probadas evidencias de beneficio neto. La franja ideológica opuesta los llama "caracoles", pues retrasan el avance y privan a pacientes de potenciales beneficios. Sin embargo hay numerosos ejemplos de grandes errores intentado acelerar los tiempos de una determinada tecnología, no siempre motivados por intereses altruistas, de la cual es muy difícil retroceder una vez que ha sido instalada poblacionalmente por mas inútil o perjudicial que resulte. Pero además, en prevención, nunca hay que perder de vista que la población blanco de un rastreo masivo son personas "sanas" (asintomáticas) a las cuales con una conducta incorrecta sólo les brindaríamos un problema. Cabe preguntarse en el caso del rastreo de cáncer de próstata: ¿Es ético ofrecer activamente a los pacientes embarcarse en una cascada de beneficio incierto y probado perjuicio, en lugar de priorizar otras acciones preventivas de probada eficacia?

Las recomendaciones de la USPSTF son seguidas por la mayoría de los grupos médicos. Sin embargo aunque en noviembre de 2009 recomendó que ya no debería ofrecerse el rastreo mamográfico en las mujeres de 40 años, salvo que este surja de una decisión informada ${ }^{9}$, tras una tormenta de controversias es probable que el rastreo en esta franja etaria no haya caído sustancialmente. La recomendación de evitar el PSA es aún más contundente ya que se aplica a los hombres sanos de todas las edades, y probablemente resultará aun más resistida. Consideremos también el escenario de algunos fabricantes de 
medicamentos y de médicos dedicados al tratamiento de los hombres con niveles elevados de PSA (dudo en estos casos llamarlos pacientes), para quienes esta actividad se ha convertido de alguna forma en un negocio lucrativo. No sería de extrañar entonces las réplicas de "batallas" políticas como las ocurridas en el seno del Congreso Norteamericano en repercusión a otras recomendaciones de la USPSTF tildadas de "racionamiento".

La gran popularidad alcanzada por la prueba de PSA desde finales de los ' 90 junto a sus consecuencias devastadoras ha llevado a Richard J. Ablin, su descubridor en 1970, ha proclamar su uso generalizado como "un desastre de salud pública"10. Desconocer que uno tenga cáncer de próstata probablemente sea hoy en día el mejor rumbo, ya que nadie vive feliz sabiéndose enfermo. Más aun si consideramos que los estudios de autopsias muestran que un tercio de los hombres de entre $40 \mathrm{y}$ 60 tienen cáncer de próstata, y que la proporción crece hasta tres cuartos después de 85 años de edad.

La evidencia de escaso o nulo beneficio e importantes perjuicios del rastreo con PSA es contundente y deja poco margen de discusión ${ }^{6}$. Tampoco hay evidencia de que el tacto rectal ni la ecografía sean eficaces. Aunque es difícil que cambie una práctica fuertemente instalada quisiera retomar la frase que abre esta editorial: "Primum non nocere". Esta locución es uno de los principales preceptos de la ética médica que se le enseña a todo estudiante de medicina. Deberíamos considerarla fuertemente al estar frente a nuestros pacientes, reconociendo que los métodos actuales no permiten ayudarlos como deseamos y así poder decirles adiós a los mismos. Sin embargo seré el primero en darle la bienvenida a nuevas tecnologías que permitan reconocer tempranamente los cánceres prostáticos de mala evolución en los que los beneficios superen con creces los perjuicios.

Recibido al 20/06/12 entregado 04/07/12

Referencias

1. USPSTF. Screening for Prostate Cancer. Current Recommendation (en línea). Disponible en URL: http://www.uspreventiveservicestaskforce.org/prostatecancerscreening.htm. Último acceso 20/6/2012.

2. Ciapponi A. Actualizaciones de cuidados preventivos de la USPSTF (3 ra edición, 2000-2003). Evid Act Pract Ambul May-Jun 2003;6(3):79-84. Disponible en URL: http://www.foroaps.org/hitalba-pagina-articulo.php?cod_producto=956 Último acceso 20/6/2012.

3. Ciapponi A. Nueva guía de cuidados preventivos de la Fuerza de Tareas de Estados Unidos (primera entrega). Evid Act Pract Ambul. 2005;8:179-182. Disponible en URL: http://www.foroaps.org/files/nueva\%20fuerza.pdf. Último acceso 20/6/2012.

4. Harris H. U.S. The New York Times. Panel Says No to Prostate Screening for Healthy Men. Disponible en URL: http://www.nytimes.com/2011/10/07/health/07prostate.html? r=2. Último acceso 20/6/2012.

5. Ciapponi A. Toma de decisiones compartidas. Evid Act Pract Ambul. Ene-Mar 2012;15(1):2-4. Disponible en URL: http://www.foroaps.org/files/toma\%20de\%20desiciones\%20compartidas.pdf. Último acceso 20/6/2012.

6. USPSTF. Screening for Prostate Cancer. Draft Recommendation Statement (en línea).

Disponible en: http://www.uspreventiveservicestaskforce.org/prostatecancerscreening/draftrecprostate.htm. Último acceso 20/6/2012.

7. Ciapponi A. Comentario Editorial: Nueva evidencia sobre el rastreo de cáncer prostático: ¿Nuevas recomendaciones? Evid Act Pract Ambul. Oct-Dic 2010;13(4):122-124. Disponible en URL: http://www.foroaps.org/files/fcgtcgfvyhol.pdf. Ultimo acceso 20/6/2012.

8. Ciapponi A. Comentario Editorial: El rastreo de cáncer de próstata y su eterno dilema: ¿es beneficioso o perjudicial? Evid Act Pract Ambul. Abr-Jun 2009;12(2):42-46. Disponible en: http://www7.foroaps.org/hitalba-pagina-articulo.php?cod_producto=2512\&origen=2. Último acceso 20/6/2012.

9. USPSTF. Screening for Breast Cancer (en línea). Disponible en: http://www.uspreventiveservicestaskforce.org/uspstt/uspsbrca.htm

10. Ablin RJ. U.S. The Great Prostate Mistake (en línea). Disponible en: http://www.nytimes.com/2010/03/10/opinion/10Ablin.html. Último acceso 20/6/2012. 